\title{
DWT Curvet based Dynamic Histogram Equalization for Brightness Preserving Contrast Enhancement of Images
}

\author{
Mukesh Kumar Barode \\ NRI Institute of Information \\ Science and Technology \\ Bhopal
}

\author{
Rajesh Kumar Rai \\ NRI Institute of Information \\ Science and Technology \\ Bhopal
}

\author{
Sachin Murarka \\ $\mathrm{NRI}$ Institute of Information \\ Science and Technology \\ Bhopal
}

\begin{abstract}
In today's world of emerging technology where most of remote sensing data has been recorded in digital formats, and almost all image interpretation and analysis involves some elements of digital processing. They may involve processing image of various measures, including coordination and correct, digital and promote to facilitate better visual interpretation, or even automated classification of targets and fully characterized by computer data. To deal with remote sensing images digitally, should be recorded and the data available in digital appropriate containers for storage in a computer disk or tape form. In this paper the image enhancement technique has been proposed for brightness preserving. Here DWT and histogram equalization has used to improve the previous result. It seems to be that the proposed method gives the better result.
\end{abstract}

\section{Keyword}

Image Enhancement, DCT, DWT

\section{INTRODUCTION}

Recent trends show that the usage of image processing has becoming more and more prominent in our daily life. In addition to television, camera, camcorder, and personal computer, many high-tech electronic products, such as handphone, or even refrigerator, nowadays are being equipped with capabilities to display digital images. Unfortunately, the input images that are provided to (or captured by) these devices are sometimes not really in good brightness and contrast. Therefore, a process known as digital image enhancement is normally required to increase the quality of these low brightness images.

Image enhancement produces an output image that subjectively looks better than the original image by changing the pixel's intensity of the input image. The purpose of image enhancement is to improve the interpretability or perception of information contained in the image for human viewers, or to provide a "better" input for other automated image processing systems.

\section{HISTOGRAM EQUALIZATION}

Suppose $\mathrm{r}$ represents the gray-levels in the image to be enhanced. For now assume that all the possible gray-levels are continuous and lie in the interval $[0 ; 1]$ (and thus $r=0$ is black and $r=1$ is white). Assume $s=T(r)$ and the following Conditions hold:

(a) $\mathrm{T}(\mathrm{r})$ is single-valued and monotonically increasing for $0 \leq$ $\mathrm{r} \leq 1$ and

(b) $0 \leq \mathrm{T}(\mathrm{r}) \leq 1$ for $0 \leq \mathrm{r} \leq 1$.

The inverse transformation $\mathrm{T}^{-1}$ is denoted by $\mathrm{r}=\mathrm{T}^{-1} 1(\mathrm{~s})$ for $0 \leq \mathrm{s} \leq 1$ (and it is assumed that $\mathrm{T}^{-1}$ also satisfies the above conditions (a) and (b)).

If we move to the domain of statistics, we can look at the gray-levels as continuous random values in the interval $[0 ; 1]$, which can be characterized by their probability density functions (PDF) pr(r) and ps(s) (and thus pr and ps are different functions). It follows that - knowing pr and $\mathrm{T}(\mathrm{r})$ and $\mathrm{T}^{-1}$ satisfies the above condition (a) - the probability density function of the transformed gray-levels can be expressed as in equation (1):

$$
\mathrm{P}_{\mathrm{S}}(\mathrm{S})=\left[\mathrm{P}_{\mathrm{r}}(\mathrm{r}) \frac{\mathrm{dr}}{\mathrm{ds}}\right] \mathrm{r}=\mathrm{T}^{-1}(\mathrm{~s})
$$

Knowing this, we now modify the appearance of the image by controlling the probability density function of its gray-levels via the transformation function $\mathrm{T}(\mathrm{r})$.Consider the transformation function, as in equation (2):

$$
\mathrm{S}=\mathrm{T}(\mathrm{r})=\int_{0}^{\mathrm{r}} \mathrm{P}_{\mathrm{r}}(\mathrm{w}) \mathrm{dw} \text { with } 0 \leq \mathrm{r} \leq 1 \ldots
$$

With w a dummy-variable needed for the integration. As in Equation (2): is also known as the cumulative distribution function (or $\mathrm{CDF}$ for short) of $\mathrm{r}$. If we take the derivative with respect to $r$ we obtain as in equation (3):

$$
\frac{\mathrm{d}_{\mathrm{s}}}{\mathrm{d}_{\mathrm{r}}}=\mathrm{P}_{\mathrm{r}}(\mathrm{r})
$$

Substituting this equation for $\frac{\mathrm{dr}}{\mathrm{ds}}$ into equation (4) is a uniform density function.

$$
\mathrm{P}_{\mathrm{S}}(\mathrm{S})=\text { with } 0 \leq \mathrm{S} \leq 1 \text {. }
$$

Note that the result is independent of the inverse transformation function. This last remark is very important since analytically obtaining $\mathrm{T}^{-1}(\mathrm{~s})$ is not always easy. Thus using a transformation function equal to the CDF of $r$ produces an image whose gray-levels have a uniform density.

The result is increases in the dynamic range of the pixels that can significantly contribute to the image's visual enhancement. Now in order to be useful in digital image processing, the previous technique must be formulated in its discrete form.

The probabilities (of the discrete gray-levels) are now with $\mathrm{L}$ the number of gray-levels in the image. The discrete form of as in equation (5) is:

$\mathrm{P}_{\mathrm{r}}\left(\mathrm{r}_{\mathrm{k}}\right)=\frac{\mathrm{n}_{\mathrm{k}}}{\mathrm{n}}$ with $0 \leq \mathrm{r}_{\mathrm{k}} \leq 1$ and $\mathrm{k}=\{0,1,2, \ldots \ldots \mathrm{L}-$ $1\} \ldots \ldots \ldots \ldots \ldots(5)$

$$
\mathrm{S}_{\mathrm{k}}=\mathrm{T}\left(\mathrm{r}_{\mathrm{k}}\right)=\sum_{\mathrm{j}=0}^{\mathrm{k}} \frac{\mathrm{n}_{\mathrm{j}}}{\mathrm{n}}
$$

$$
=\sum_{\mathrm{j}=0}^{\mathrm{k}} \mathrm{P}_{\mathrm{r}}\left(\mathrm{r}_{\mathrm{j}}\right) \text { with } 0 \leq \mathrm{r}_{\mathrm{k}} \leq 1 \text { and } \mathrm{k}=
$$

$\{0,1,2, \ldots \ldots . L-1\}$.

The inverse transformation is denoted by $\mathrm{r}_{\mathrm{k}}$.

$$
\mathrm{r}_{\mathrm{k}}=\mathrm{T}^{-1}\left(\mathrm{~S}_{\mathrm{k}}\right) \text { with } 0 \leq \mathrm{S}_{\mathrm{k}} \leq 1
$$


The transformation function $\mathrm{T}\left(\mathrm{r}_{\mathrm{k}}\right)$ can be computed directly from the image by using equation (8). The main advantage of this technique (histogram equalization) is that pictures with a very poor dynamic range (thus having very small variations in their gray Levels can be enhanced such that all their original gray-levels are 'smeared out' in the spectrum from black to white (note that white is always reached).

This leads to more visible details in the output image. A disadvantage of this method is that visual artifacts can be introduced resulting in graininess and patchiness. To retrieve the gray-levels of $\mathrm{Z}$, we apply the inverse process as in equation (9):

$$
\mathrm{Z}=\mathrm{G}^{-1}(\mathrm{~V}) \text {. }
$$

Now remember that these gray-levels $\mathrm{Z}$ are precisely the gray-levels we are searching for because histogram equalization always results in the same uniform histogram, $\mathrm{P}_{\mathrm{S}}(\mathrm{S})$ and $\mathrm{P}_{\mathrm{v}}(\mathrm{V})$ are equal. If, in the inverse process, we now use the uniform gray-levels $\mathrm{s}$ obtained from the original image instead of using v, the resulting Gray-levels would have the desired probability density function.

$$
\mathrm{Z}=\mathrm{G}^{-1}(\mathrm{~s})
$$

If $\mathrm{G}^{-1}$ (s) is single-valued, as in equation (10): we can summarize as follows:

Equalize the gray-levels of the original image, Specify the desired probability density function and obtain the transformation function using equalization on the desired image.

3. Apply the inverse transformation function to the graylevels obtained in the first step.

The result is an output image with its gray-levels characterized by a certain specified probability density $\mathrm{P}_{\mathrm{Z}}(\mathrm{Z})$.Note that two transformation functions are needed with this technique (namely first $\mathrm{T}^{\mathrm{r}}$ and then $\mathrm{G}^{-1}$ (s), they can be simply combined into one single Function: Note that although as in equation (11) is rather easily applied to discrete variables, it can become a pain staking task when dealing with continuous variables, because then the results have to be derived analytically.

$$
\begin{gathered}
\mathrm{Z}=\mathrm{G}^{-1}(\mathrm{~S}) \Rightarrow \mathrm{Z}= \\
\mathrm{G}^{-1}(\mathrm{~T}(\mathrm{r})) \ldots \ldots \ldots \ldots \ldots \ldots \ldots \ldots \ldots \ldots \ldots \ldots \ldots \ldots \ldots \ldots \ldots \ldots \ldots \ldots
\end{gathered}
$$

Haidi Ibrahim and Nicholas Sia Pik Kong, "Brightness Preserving Dynamic Histogram Equalization for Image Contrast Enhancement”, IEEE 2007, pp 1752-1758.

Histogram equalization (HE) or Equalization graph is one of the most common methods used to enhance contrast in digital images. However, this technique is not exactly a very suitable to be implemented in the field of consumer electronics, such as TVs, because the method tends to introduce unnecessary, such as the effect of visual impairment saturation. One of the solutions to overcome this weakness is to keep the average brightness of the input image in the output image. This paper proposes a new method, known as the brightness to maintain the dynamic graphic equation, which is an extension of happiness that can produce the output image with the means of almost equal intensity to the average density of the input, and achieve conservation requirements of the average brightness of the image. First, how to input graph dimensional Gaussian smoothing filter, and then divide the graph, paving the maximum local base. After that, each section is adjusted to the new dynamic range. After that, irrespective of the graphic application process equation for these sections, based on this new dynamic range. Certainly, changes in the dynamic range, and also change the equation Figure process the average brightness of the image. Therefore, the final step in this method is to normalize the image output to the average brightness of the input. Our results of 80 test images show that this method is superior to the average brightness to keep the graphic and other current methods equation. In most cases, successful BPDHE enhance the image without serious side effects, while at the same time keeping the average brightness of the input and use different types of methods to improve contrast. Maintain brightness and noise reduction is also an improvement of photo techniques. Some methods of either the spatial domain or domain method using the method proposed frequency diverse applications such as conversion modes.

Not all conversion methods are suitable for a particular application. The suitability of the method depends on the type of image processing or transformations applied to the image, and reduce noise of the type used to enhance the image. The objective and quantitative measure of the suitability of each method for a particular application is of great interest and importance.

\section{PROPOSED APPROACH}

The research described in this dissertation focuses on the field of brightness preservation, contrast enhancement and reducing noise technique of image enhancement. It is clear that the last condition for processing digital images is a computer system, sometimes referred to as an image analysis system with appropriate hardware and software for data processing. Many of the available software systems developed specifically for commercial remote sensing and image processing and analysis.

The IDBPHE method was preserves image brightness and enhances visualization of images more effectively, but it depends on the size of image and result obtained in gray level. They have used only two parameters PSNR and AMBE then compare with various previous methods. Histogram equalized images uses full dynamic range of the pixel values for maximum contrast. However, this sometimes does not give better visual quality.

The flow graph also explains the proposed approaching deep. Here the input image has been used on which the histogram will apply. HE applied image will face the step of DWT. Here haar transformation technique has used. It will generate four types of images. . in this approach LL has used for better results.After that the DCT has been applied in the LL part of an image. The whole process is shown in flow graph. 


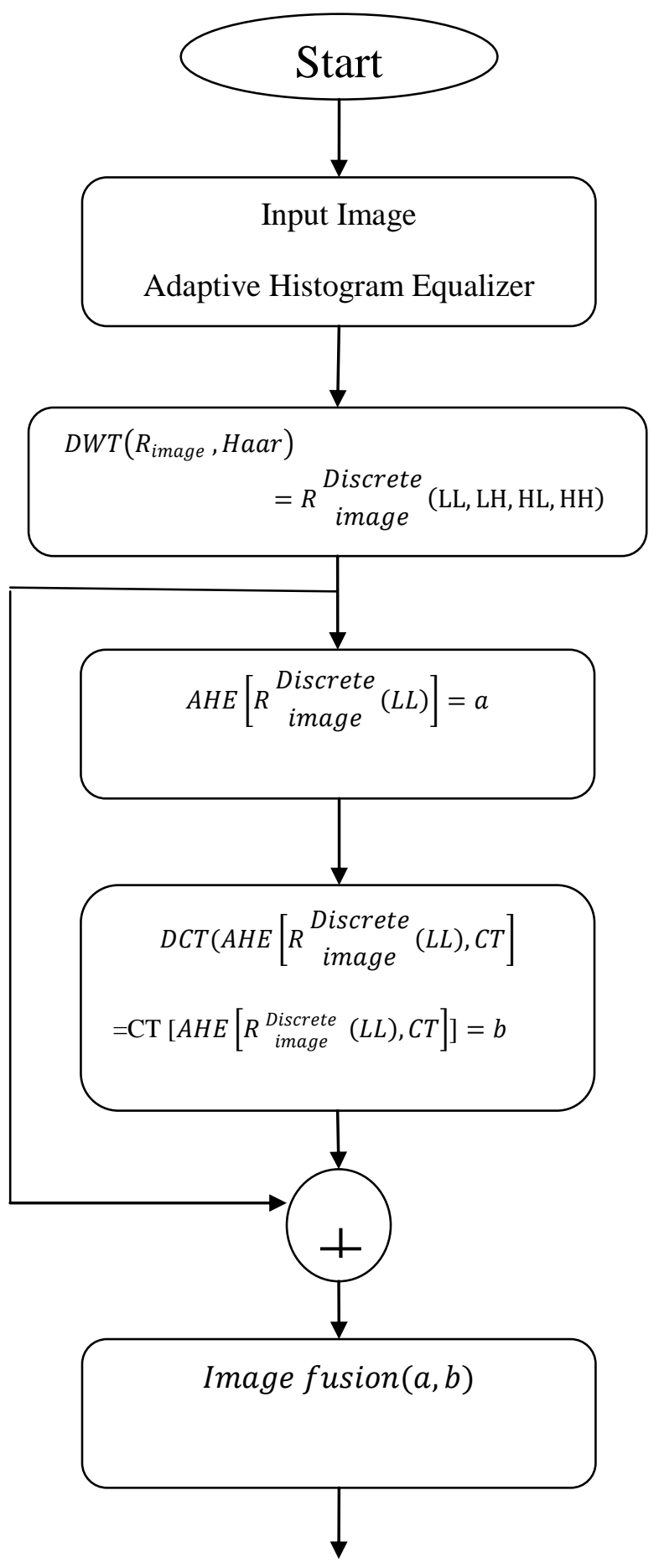

Figure 1:- Proposed Work

\section{EXPERIMENTAL RESULTS}

The implementation of the proposed work has done in Matlab 7.5.0.342(R2007b) software platform has used to perform the environments. The laptop for experiment is equipped with a windows 7 home basic with $2.50 \mathrm{Ghz}$, Intel (R) core(TM) i5$3210 \mathrm{M}$ processor with installed memory RAM 4 GB on 64 bit operating system.

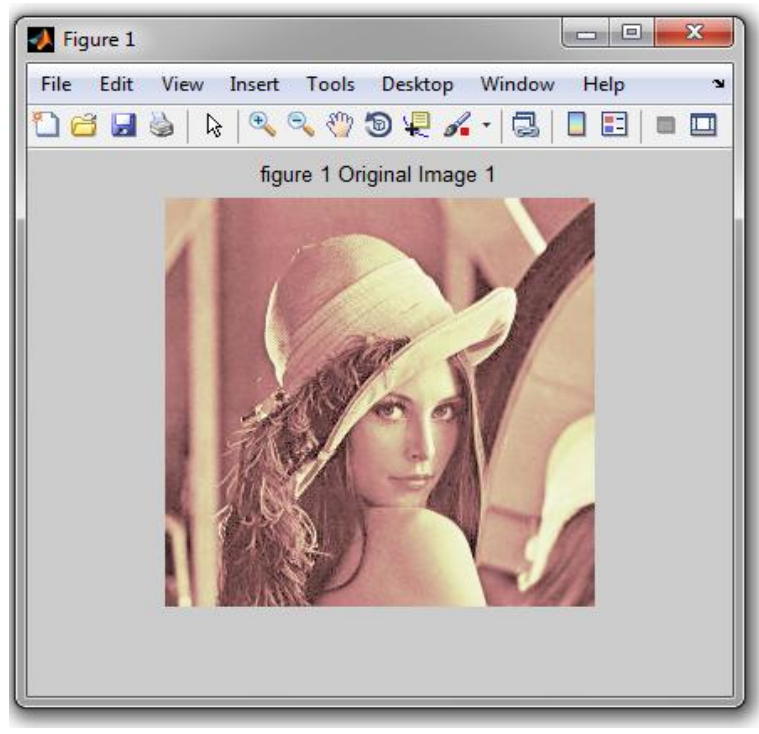

Figure 2: Original input image

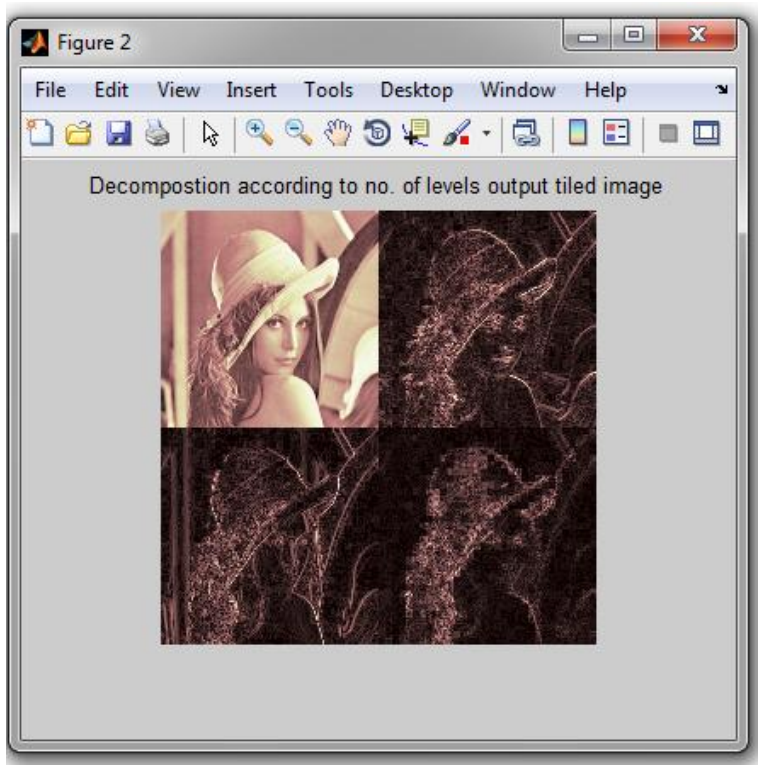

Figure 3: Decomposed Images

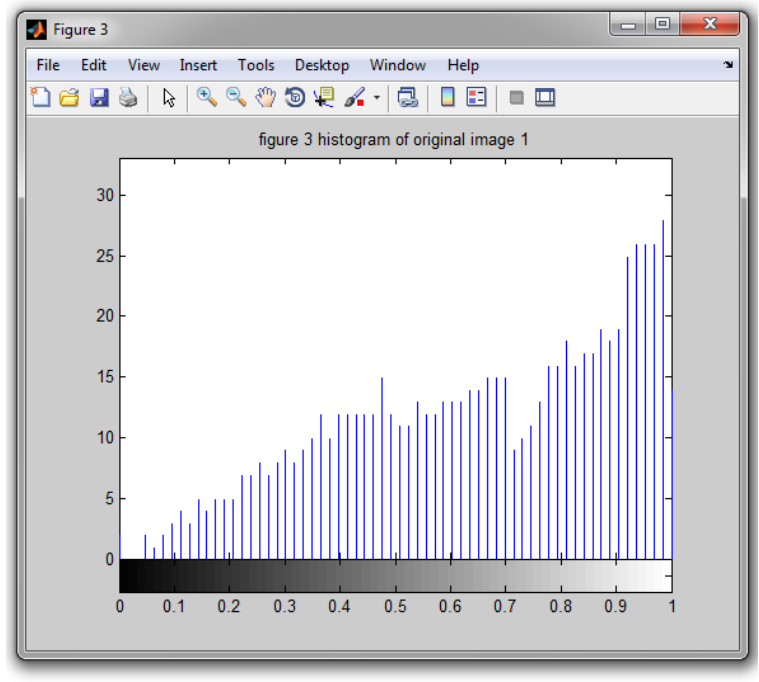

Figure 4: Histogram of input image 


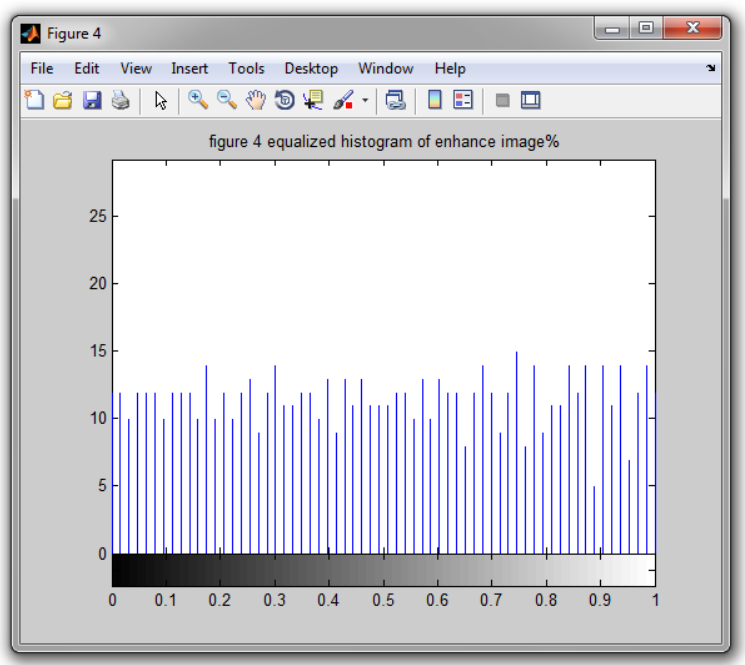

Figure 5: Histogram after Equalization

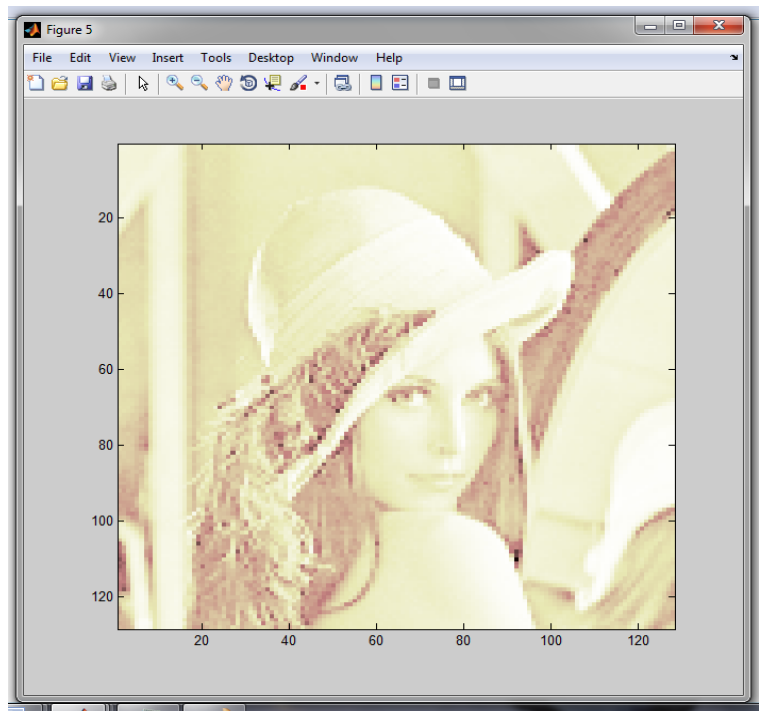

Figure 6: Histogram Equalized Image

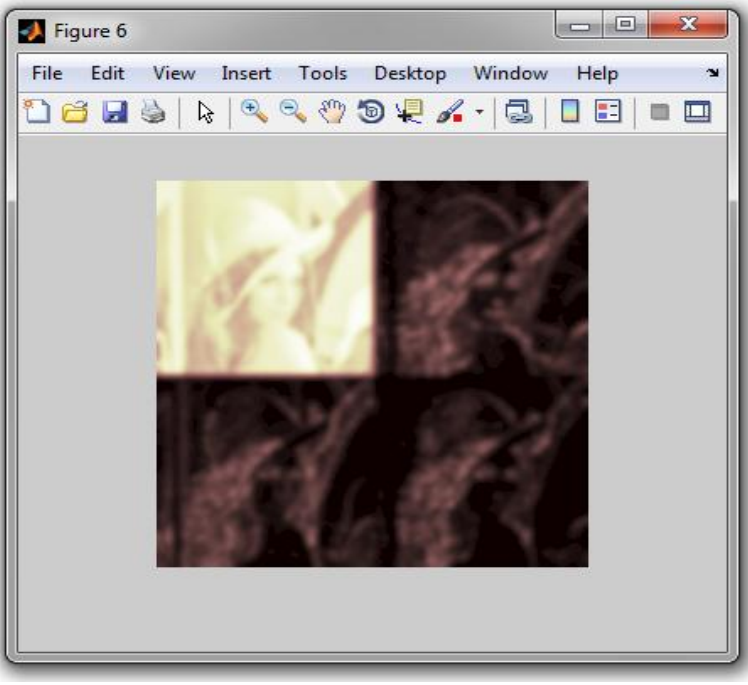

Figure 7: Decomposed after Equalization

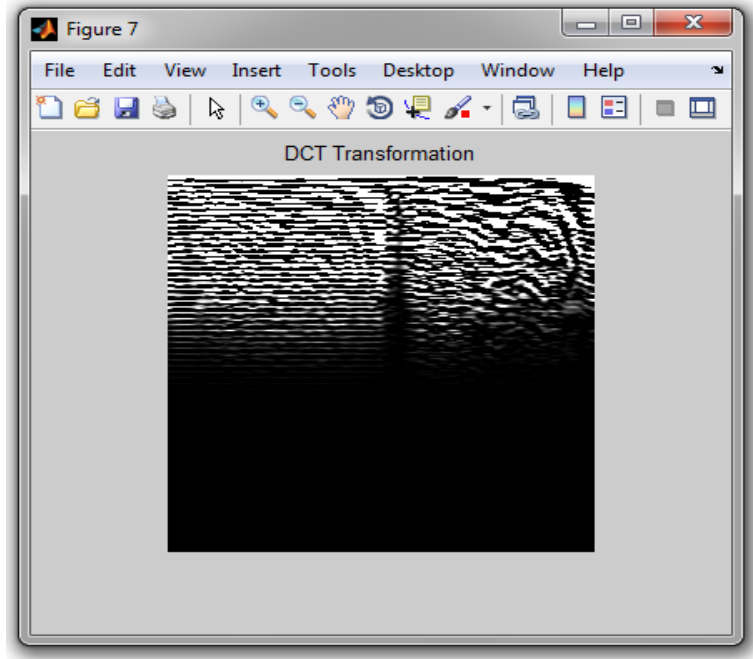

Figure 8: DCT Transformation

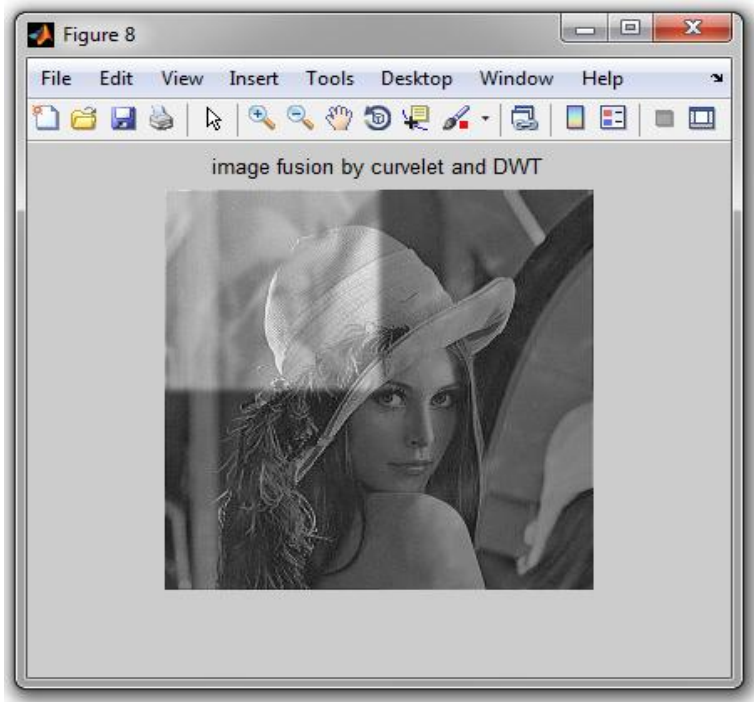

Figure 9: Image Fusion

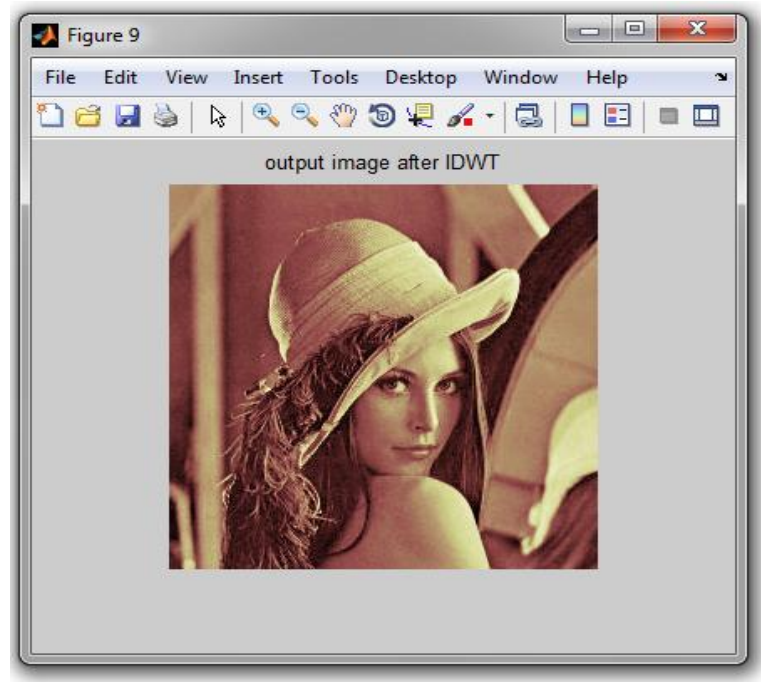

Figure 10: Output Image 
Assuming that $\mathrm{M} * \mathrm{~N}$ is the total no of pixel in the input or output image, MSE is calculated through the equation below, based on MSE,PSNR is then defined as .

$$
\text { MSE }=\frac{\sum_{\mathrm{M}, \mathrm{N}}\left[\mathrm{I}_{1}(\mathrm{~m}, \mathrm{n})-\mathrm{I}_{2}(\mathrm{~m}, \mathrm{n})\right]^{2}}{\mathrm{M} * \mathrm{~N}}
$$

PSNR is calculated using the following equation

$$
\mathrm{PSNR}=10 \log _{10}\left(\frac{\mathrm{MAX}^{2}}{\mathrm{MSE}}\right)
$$

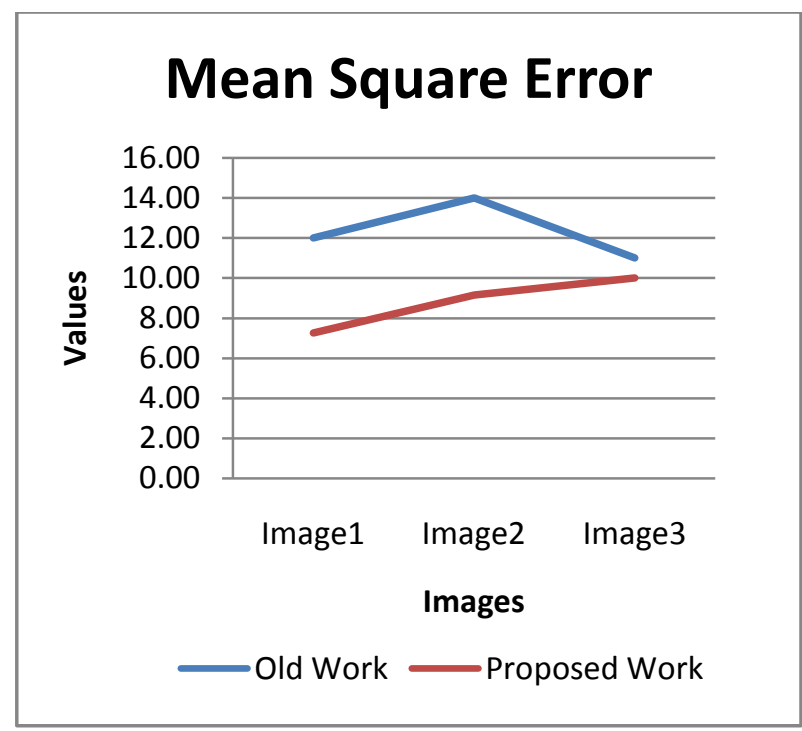

Figure 11:- Graph to Show MSE

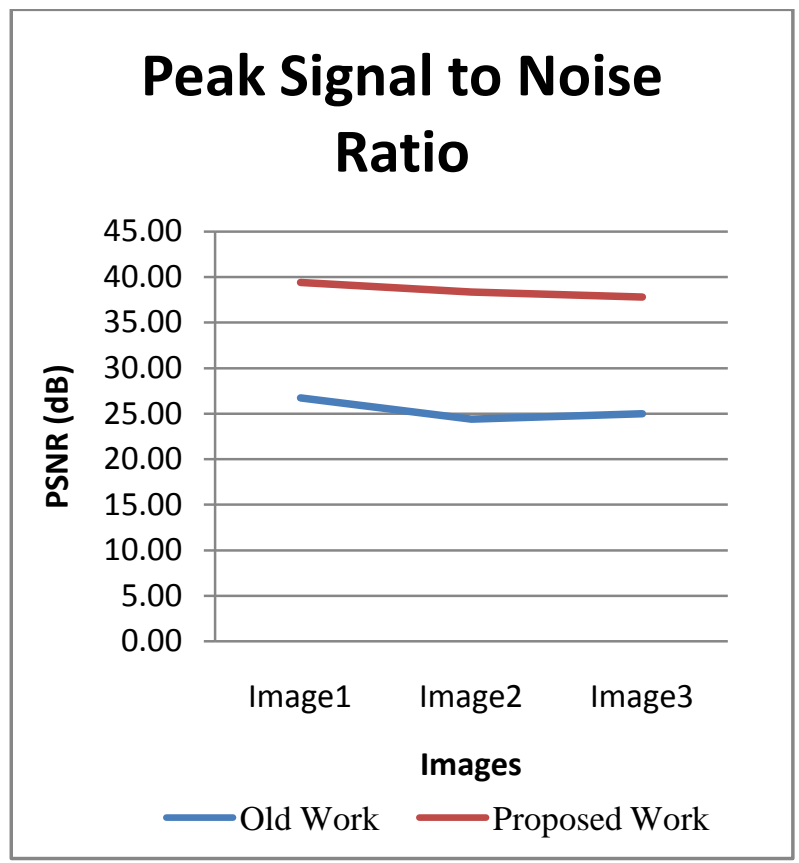

Figure 12:- Graph to Show PSNR

\section{CONCLUSION}

This Work has presented here, a new hybrid transformation model based on DCT, DWT and image fusion. For preserving brightness, contrast enhancement and reducing noise for high brightness and low contrast images. Performance of this technique has been compared with Old work. The experimental result show that the proposed method gives better performance in terms of brightness and contrast of the enhanced image. Thus this model can be consider, suitable for preserving brightness and reducing noise for low contrast and high brightness medical images.

\section{REFERENCES}

[1] D. Menotti, L. Najman, J. Facon and A. A. Araujo, "Multi-Histogram Equalization Methods For Contrast Enhancement And Brightness Preservation", IEEE Trans. Consumer Electron., vol. 49, no.4, pp. 1301-1309, November 2003

[2] H.Ibrahim and N. S. P. Kong, "Brightness Preserving Dynamic Histogram Equalization For Image Contrast Enhancement", IEEE 2007, volume. 53, no. 4, pp. 1752 1758.

[3] Nyamlkhagva Sengee and Heung Kook Choi, "Brightness Preserving Weight Clustering Histogram Equalization", IEEE Trans. Consumer Electron. vol. 54, no. 3, pp. 1329-1337, August 2008.

[4] Hojat Yeganeh, Ali Ziaei and Amirhossein Rezaie, "A Novel Approach For Contrast Enhancement Based On Histogram Equalization", In Proceedings of the International Conference on Computer and Communication Engineering,vol.3,no.4, pp. 256260 ,febury 2008

[5] J. Tang, E. Peli and S. Acton, "Image Enhancement Using A Contrast Measure in the Compressed Domain", IEEE 2003 volume. 10, no. 10, pp. 289-292.

[6] N. Sengee and H. K. Choi "Brightness Preserving Weight Clustering Histogram Equalization", IEEE Transactions 2008, Volume. 54, No. 3,

[7] Mary Kim and Min Gyo Chung, "Recursively Separated and Weighted Histogram Equalization for Brightness Preservation and Contrast Enhancement", IEEE 20008 Volume 54, no. 3, pp. 1389-1397,

[8] Chao Wang and Zhongfu Ye, "Brightness Preserving Histogram Equalization with Maximum Entropy A Variational Perspective", IEEE 2005 , Volume 51, no. 4, pp. 1326-1334

[9] Md. Foisal Hussein and Mohammad Reza Alsharif, "Minimum Mean Brightness Error Dynamic Histogram Equalization For Brightness Preserving Image Contrast Enhancement", IJICIC 2009, Volume 5, no. 10, pp. 3249-3260

[10] Wang Zhiming, TAO Jianhua, "A Fast Implementation of Adaptive Histogram Equalization", ICSP 2006 Proceedings, IEEE January 2006.

[11] Stephen M. Pizer, R. Eugene Johnston, James P. Erickson, Bonnie C.N Yankaskas, Keith E. Muller, "Contrast-Limited Adaptive Histogram Equalization Speed and Effectiveness", IEEE Int. Conf. Neural Networks \& Signal Processing, Nanjing, China, pp.1417, December 2003.

[12] Chung-Cheng Chiu, Sheng-Yi Chiu, Han-Ni Yang, and Ching-Tung Lo "Histogram Enhancement Using Adaptive Segmentation Algorithm", MVA IAPR Conference on Machine Vision Applications,Nara,Japan,pp.13-15, June 2011. 